\title{
INFLUÊNCIA DOS FATORES CONSCIÊNGIA AMBIENTAL E HÁBITOS DE CONSUMO SUSTENTÁVEL SOBRE A INTENÇÃO DE COMPRA DE PRODUTOS ECOLÓGICOS DOS INDIVIDUOS
}

INFLUENCE OF FACTORS ENVIRONMENTAL CONSCIOUSNESS AND SUSTAINABLE CONSUMPTION HABITS ON THE INTENTION TO BUY ECOLOGICAL PRODUCTS FROM INDIVIDUALS

\section{Vanessa Edy Dagnoni Mondini}

Instituto Federal de Santa Catarina (IFSC), Florianópolis/SC, BRASIL

prof.vanessa@ymail.com

\section{Gustavo da Rosa Borges}

Universidade Regional de Blumenau (FURB), Blumenau/SC, BRASIL

profevanessamondini@gmail.com

\section{Luis Cesar Mondini}

Universidade Regional de Blumenau (FURB), Blumenau/SC, BRASIL

cesar.mondini@yahoo.com.br

\section{Marialva Tomio Dreher}

Universidade Regional de Blumenau (FURB), Blumenau/SC, BRASIL

marialvatomio@yahoo.com.br

\begin{abstract}
Resumo
A responsabilidade sobre questões ecológicas, anteriormente atribuída às esferas pública e privada, vem se estendendo cada vez mais para os hábitos e comportamentos de consumo da sociedade e, em especial, dos indivíduos. Sob esta ótica, o objetivo deste estudo é analisar a influência dos fatores consciência ambiental e hábitos de consumo sustentável sobre a intenção de compra de produtos ecológicos dos indivíduos. Nesta perspectiva, realizou-se uma pesquisa quantitativa e descritiva com 182 estudantes de uma instituição de ensino superior catarinense. Utilizou-se como instrumento de coleta de dados, um questionário estruturado, baseado nos estudos de Cardoso e Cairrão (2007). A análise dos dados foi realizada por meio da modelagem de equação estrutural. Os resultados obtidos indicaram que a intenção de compra de produtos ecológicos é maior quando o consumidor é mais consciente sobre questões ambientais.
\end{abstract}

Palavras-chave: Consciência Ambiental. Hábitos de Consumo Sustentável. Intenção de Comprar Produtos Ecológicos.

\begin{abstract}
Responsibility for ecological issues, previously attributed to the public and private spheres, has been increasingly extended to the consumption habits and behavior of society and, in particular, of individuals. From this perspective, the objective of this study is to analyze the influence of environmental awareness factors and sustainable consumption habits on the individuals' intention to purchase ecological products. In this perspective, a quantitative and descriptive research was carried out with 182 students from a higher education institution in Santa Catarina. A structured questionnaire, based on the studies of Cardoso and Cairrão (2007), was used as a data collection instrument. Data analysis was performed using structural equation modeling. The results indicate that the intention to buy organic products is greater when the consumer is more aware about environmental issues.
\end{abstract}

Keywords: Environmental Awareness. Habits of Sustainable Consumption. Intent to Buy Ecological Products. 


\section{Introdução}

Questões relacionadas à produção limpa, uso eficiente dos recursos e tratamento de resíduos pós-consumo se tornaram pauta frequente em discussões ambientais nas últimas décadas (FERREIRA et al., 2015). Dentro destas temáticas, o comportamento de consumo passou a ser cada vez mais incorporado pela arena pública de ativismo, e os consumidores, encorajados a fazer a sua parte adquirindo apenas produtos considerados verdes, sustentáveis ou ambientalmente amigáveis (DEFRA, 2003).

Promover o consumo sustentável implica em enfrentar questões delicadas que envolvem os princípios de soberania e hábitos do consumidor, a determinação das quantidades de consumo suficientes e a visão cultural da felicidade atrelada à prosperidade material (MONT; PLEPYS, 2008). Apesar da ainda pouca especificidade relacionada ao tema, é certo que os maiores problemas ambientais decorrem da inadequação dos padrões de consumo contemporâneos (SCHULTZ, 2002).

O desenvolvimento sustentável implica em satisfazer as necessidades atuais sem comprometer o atendimento das necessidades das gerações futuras (MASERA, 2001; DIAS, 2013). Esta relação de adaptação e harmonia entre a humanidade e a natureza, no entanto, nem sempre obtém respaldo, especialmente quando a visão de que a natureza é algo a ser conquistado, prevalece (KUHLMAN; FARRINGTON, 2010).

A responsabilidade sobre questões ecológicas, anteriormente atribuída às esferas pública e privada, vem se estendendo cada vez mais para os hábitos e comportamentos de consumo da sociedade e, em especial, dos indivíduos. Atualmente, já é consenso que não apenas governos e empresas negligentes são responsáveis pela relação desastrosa do homem com o meio ambiente, mas o indivíduo também (PORTILHO, 2005).

Mesmo que nem sempre por motivos altruístas, afinal a degradação da natureza impacta negativamente na sobrevivência do próprio homem, percebe-se que as pessoas passaram a valorizar as questões ambientais e a adotar comportamentos mais sustentáveis em sua rotina (OTTMAN, 1993; CALOMARDE, 2000; LAMBIN, 2002; PORTILHO, 2005; KRUTER; BARCELLOS; SILVA, 2012). Quando o comportamento em prol do meio ambiente se alinha ao interesse próprio, os indivíduos tendem a cumpri-lo (AJZEN, 1991). Observa-se que aos poucos, além da pressão exercida pela sociedade para que governos e organizações se voltem a questões ecológicas, os comportamentos individuais irresponsáveis também vêm sofrendo represálias (PORTILHO, 2005).

No entanto, os movimentos sociais em prol do meio ambiente parecem se concentrar em temas relacionados à preservação, não poluição e reciclagem (CONNOLLY; PROTHERO, 2003). Quando as ações exigem que o ser humano altere seus hábitos de consumo, no entanto, vários estudos indicam que a adesão à causa é menos intensa (HORNE, 2009, YOUNG et al., 2010). Apesar de os consumidores se dizerem propensos a comprar de forma sustentável, estas declarações nem sempre respaldam suas práticas cotidianas (OECD, 2005; PEDERSEN; NEERGAARD, 2006), tornando o discurso da sustentabilidade vazio (VEIGA, 2006).

O consumo é entendido como um indicador de qualidade de vida. Isto gera problemas, já que o crescimento dos níveis de consumo está diretamente relacionado à degradação ambiental (MONT; PLEPYS, 2008). Dentre os consumidores, o público jovem costuma ser o mais associado ao materialismo e ao consumismo. No entanto, quando este perfil é composto por universitários, presumese que, por estarem cercados de informações sobre questões atuais, em que o tema sustentabilidade se insere, eles estejam mais positivamente esclarecidos sobre as questões do consumo sustentável (CARDOSO; CAIRRÃO, 2007).

A percepção do público universitário sobre questões ambientais já permeou diversos estudos (CARDOSO; CAIRRÃO, 2007; GORNI, GOMES; DREHER, 2012; MONTEIRO et al., 2012), mesmo assim, Marchand e Walker (2008) destacam que os estudos a respeito do consumo sustentável ainda são inconclusivos.

Buscando uma compreensão da percepção sobre questões ambientais junto a alunos de uma universidade catarinense, este estudo tem como objetivo analisar a influência da consciência ambiental e dos hábitos de consumo sustentável sobre a intenção de compra de produtos ecológicos.

Pouco se sabe sobre como a sustentabilidade do produto afeta as preferências dos consumidores (LUCHS, 2010). Este estudo favorecerá que se conheçam por meio de um estudo empírico, os preditores capazes de influenciar a intenção de compra de produtos ecológicos possibilitando a identificação e implementação de estratégias que possam melhor influenciar a mudança de atitude por parte dos consumidores. Entender os fatores que potencialmente influenciam a percepção dos consumidores pode favorecer uma compreensão adicional das intenções de compra (D'SOUZA, 2006). 


\section{Fundamentação Teórica}

Nesta etapa será apresentada a revisão da literatura a respeito da intenção de compra de produtos ecológicos, da consciência ambiental e dos hábitos de consumo sustentável. Além disso, serão expostos os resultados de estudos anteriores relacionados ao tema.

\section{Intenção de Compra de Produtos Ecológicos}

Pautas relacionadas ao consumo sustentável movimentam a agenda política dos países desde o final do século XX (COHEN, 2001; RUMPALA, 2011), resultando em um consenso sobre a alta capacidade de esgotamento e degradação ambiental promovida por modelos consumistas de desenvolvimento econômico e social (CARVALHO, 2001; CHEN; CHAI, 2010). No entanto, perspectivas de consumo sustentável ainda não se consolidaram em função das mudanças de comportamentos e estilos de vida exigidos.

O simples ato de comprar produtos que não agridam o meio ambiente, não isenta o consumidor das responsabilidades ambientais. Produtos alimentícios e os movidos à energia são considerados mais críticos do ponto de vista da sustentabilidade (TUKKER et al., 2010). É preciso associar qualidade de vida a aspectos menos materiais (MONT; PLEPYS, 2008) como o consumo cultural e social e estilos de vida sustentáveis que promovam experiências de sustentabilidade partilhadas entre famílias, grupos e comunidade (SCHAEFER; CRANE, 2005).

Embora o interesse público em temas relacionados ao consumo esteja aumentando, o comportamento efetivo dos consumidores ainda não acompanhou esta tendência (VERMEIR; VERBEKE, 2006) e critérios como preço e conveniência são ainda muitas vezes considerados como mais importantes (ROBERTS, 1996).

Em parte, mudanças comportamentais, em função de sua multiplicidade de razões e influências, costumam levar tempo para acontecer, especialmente em sociedades em que a orientação para o consumo é tão difundida (MOISANDER, 2007). Por outro lado, o próprio processo de aquisição de produtos ecológicos é complexo em função da interação de inúmeras variáveis como preço, consciência, confiança, disponibilidade de informações, reputação da marca entre outros (MANZINI et al., 2006).

O entendimento do comportamento de compra demanda a análise dos processos intrínsecos aos momentos de seleção, aquisição e uso de produtos pelos consumidores (SOLOMON, 2002). Influenciam este comportamento, características individuais, ambientais e psicológicas difíceis de serem identificadas (ENGEL; BLACKWELL; MINIARD, 2000). Apesar de muitos consumidores afirmarem levar em conta características pró-ambientais em suas aquisições, a maioria ainda considera o preço e a comodidade como critérios norteadores das compras (HORNE, 2009).

A demanda por produtos ecológicos é ainda uma tendência recente e sugere que a integração de atributos não econômicos ao produto também pode agregar valor a ele (BRÉCARD et al, 2009). Produtos considerados ecológicos são caracterizados como não poluentes, não tóxicos, favoráveis ao meio ambiente e à saúde das pessoas (WALDMAN; SCHNEIDER, 2000). Seu desenvolvimento ocorre a partir da escolha de matérias-primas renováveis, recicláveis e que conservem os recursos naturais no momento de extração. $O$ processo também deve prever o uso eficiente da água, energia e solo, o correto descarte ou reutilização (CALOMARDE, 2000) e condições que favoreçam a redução ou a reutilização de embalagens, além do aumento da vida útil dos produtos e a possibilidade de sua transformação em adubo (LAMBIN, 2002).

Consumir um determinado produto devido ao seu impacto ambiental positivo percebido pode ser descrito como um comportamento ecológico (BRÉCARD et al., 2009). Estudos relacionados à intenção de compra de produtos ecológicos ainda se mostram inconclusivos quanto à postura dos consumidores. Enquanto alguns já identificaram atitudes favoráveis ao ambiente (VERMEIR; VERBEKE, 2006; MOTTA; OLIVEIRA, 2007; SILVA, FERREIRA; FERREIRA, 2009, MONTEIRO et al, 2012; NAGAR, 2013; WIEDMANN et al., 2014), outros apontaram posturas de indiferença ou de despreocupação dos consumidores (YOUNG et al., 2010; VIEIRA, 2013).

Vermeir e Verbeke (2006) investigaram a diferença entre a atitude favorável em relação ao comportamento sustentável e intenção comportamental para adquirir produtos alimentares sustentáveis junto a 456 jovens consumidores. Observaram que a consciência ambiental tem um impacto positivo na atitude em relação à compra de produtos sustentáveis. Apesar disso, o consumo de produtos sustentáveis é influenciado por normas sociais e disponibilidade do produto.

Motta e Oliveira (2007) estudaram o interesse dos consumidores em comprar produtos considerando critérios ecológicos. Verificaram que estes consumidores se mostraram propensos a pagar mais por produtos ambientalmente amigáveis, indicando que se confere maior valor a esses produtos. 
Silva, Ferreira e Ferreira (2009) avaliaram a influência de estratégias de marketing verde sobre o comportamento de 272 consumidores de São Paulo e verificaram que a divulgação de produtos verdes nas lojas, influencia a intenção de compra dos clientes.

Monteiro et al (2012) mediram o nível de consciência ecológica de 150 alunos de administração de uma IES de São Paulo e verificaram que estes estudantes já demonstram interesse nas questões ambientais.

Nagar (2013) pesquisou 180 consumidores de produtos verdes e identificou uma propensão deste público a pagar mais por produtos ecológicos, além de apresentarem maior lealdade a estas marcas. Conforme o pesquisador, produtos e serviços divulgados como ecológicos são percebidos como mais seguros de usar, conferindo à empresa maior credibilidade.

Em contrapartida, o estudo de Marchand e Walker (2007) sobre consumo responsável e as preferências dos consumidores, indicou que as pessoas adotam estilos de vida mais sustentáveis não só por causa de uma consciência ecológica, mas também por causa de fatores ou benefícios pessoais percebidos.

Young et al. (2010) ao pesquisarem sobre hábitos de consumo, perceberam uma lacuna entre a atitude afirmada e o comportamento exercido pelos entrevistados. Muitos dos consumidores pesquisados se disseram preocupados com questões ecológicas, no entanto, este critério nem sempre é levado em conta no momento da efetivação das compras.

Vieira (2013) identificou o interesse de 261 consumidores de supermercados da cidade de Porto Alegre, na compra de alimentos orgânicos. Apesar disso, as razões apresentadas ainda não se referem a consciência ambiental e sim, a crenças sobre os benefícios à saúde ou status que este tipo de produto proporciona.

De maneira geral, mesmo que por motivos diferentes, parece haver um entusiasmo coletivo sobre questões ambientais, o que acaba gerando um aumento na demanda por produtos qualificados como orgânicos, verdes ou ecológicos. Estes produtos são entendidos pelos consumidores como superiores em relação ao sabor, qualidade, segurança, impacto na saúde e no meio ambiente, resultando em uma pré-disposição a pagar mais e a recomendar estes produtos a terceiros (WIEDMANN et al., 2014).

\section{Consciência Ambiental}

Alardes relacionados a alimentos contaminados, depredação da natureza, perda de biodiversidade, alterações climáticas, poluição química e outros riscos ambientais e de saúde vem permeando o cotidiano das pessoas (BOSTRÖM; KLINTMAN, 2008). Em tese, estas ameaças promovem maior consciência ambiental e deveriam ser decisivas no momento da aquisição de produtos ecologicamente corretos (CALOMARDE, 2000). No entanto, consumidores ambientalmente conscientes não necessariamente possuem comportamentos ambientalmente sustentáveis (OHTOMO; HIROSE, 2007).

O número de consumidores que se dizem conscientes sobre os problemas ambientais vem crescendo (FRAJ; MARTINEZ, 2006). A conscientização ambiental implica em mudança de comportamento e envolve essencialmente uma questão de educação (BUTZKE; PEREIRA; NOEBAUER, 2001). Problemas ambientais só podem ser combatidos pela conscientização, a partir de processos educacionais que envolvem a mudança de atitudes da família, no local de trabalho, instâncias públicas e privadas, nacionais e internacionais (FURRIELA, 2001).

Mudanças de atitudes são realizadas com relação a algum aspecto do mundo do indivíduo e refletem a sua avaliação do problema em questão em termos de bom ou mau, maléfico ou benéfico, agradável ou desagradável (AJZEN; FISHBEIN, 1980; AJZEN, 1991). O desenvolvimento da consciência ambiental se inicia nos valores aprendidos ao longo da vida sobre benefícios e prejuízos ambientais causados pelos seres humanos (DIAS, 2013) e influenciada pelos relacionamentos interpessoais (ANDERSON Jr.; CUNNINGHAM, 1972).

Consciência ambiental indica a utilização dos recursos ambientais de forma sustentável ou que o consumo esteja atrelado ao que se consegue produzir sem prejudicar o ambiente para as gerações futuras. Consumidores ambientalmente conscientes baseiam suas decisões em critérios de sustentabilidade e assumem responsabilidade por suas escolhas de consumo (WEBSTER, 1975). Ao vincularem responsabilidade ambiental a seus padrões de consumo, os indivíduos transformam seu foco e passam a consumir produtos com menor impacto à natureza (MOTTA; ROSSI, 2003). A compra de produtos ecológicos indica que os consumidores possuem valores consoantes às questões ecológicas atuais.

Modificações de comportamento exigem mudanças sociais e institucionais que estimulem comportamentos de consumo ambientalmente mais saudáveis (GOODWIN et 
al., 1997). É preciso instituir políticas culturais de estímulo ao consumo sustentável tanto em nível acadêmico quanto político (HOBSON, 2003).

Fatores sociais, institucionais, ausência de estímulos governamentais e falta de clareza sobre os impactos ambientais decorridos das escolhas individuais de compra, dificultam a mudança de hábitos, mesmo nos casos em que o conhecimento e a compreensão sobre o tema estejam presentes (MONT; PLEPYS, 2008).

A consciência ambiental colabora para a redução dos hábitos de desperdício (PINTO et al., 2011). A ampliação do consumo de produtos ecológicos implica em maior conhecimento dos consumidores a respeito de questões ambientais e do desenvolvimento de uma consciência ambiental. A partir de então, será possível a adoção de atitudes e comportamentos ambientalmente responsáveis. Partindo deste princípio, estratégias que enfatizem programas educacionais e pesquisas sobre o tema, são essenciais (PORTILHO, 2005).

Os valores pessoais são moldados por meio de experiências e processos de aprendizagem. Indivíduos que demonstram consciência ambiental expressam esta postura adotando atitudes positivas em relação à compra de produtos ecológicos, (KAHLE, 1996). A perspectiva de adotar um comportamento politicamente correto e assim sentir-se legitimado perante o grupo leva o indivíduo, mesmo que de forma inconsciente, a hábitos ecologicamente amigáveis (GARCIA, 2008).

Consumidores ambientalmente conscientes ponderam as consequências do seu consumo e busca influenciar outras pessoas (WEBSTER, 1975). As decisões de compra são claramente influenciadas pelo aumento da consciência ambiental (MENON; MENON, 1997; PRIEWASSER, 1999). O conhecimento ambiental tem sido frequentemente considerado como o principal motivador do comportamento do consumidor verde (PEATTIE, 2010). Se o comportamento do consumidor é influenciado por crenças, valores e experiências é preciso que a consciência ambiental seja despertada desde cedo (MATTAR, 2013). Atualmente, há a disponibilidade de inúmeras ferramentas tecnológicas de informação e comunicação que podem ser usadas para promover ações educativas e um maior engajamento à causa ambiental. Aumentar o nível de consciência ecológica contribui para que o consumidor se preocupe com o impacto ambiental causado por seus atos (BEDANTE; SLONGO, 2004).

A consciência ambiental é tão importante, que Vermeir e Verbeke (2006) a apontaram como influenciadora de atitudes positivas em relação à compra de produtos sustentáveis ou opções menos agressivas à natureza; podendo ainda, ser a principal propulsora para que exista a compra sustentável (WEBSTER, 1975; MENON; MENON, 1997; PRIEWASSER, 1999; PEATTIE, 2010).

Pinto et al. (2011) ainda apontam que a consciência ambiental colabora para a redução dos hábitos de desperdício. Marchand e Walker (2007), no entanto, sugerem que além da consciência ecológica, fatores ou benefícios pessoais percebidos nos produtos ecológicos também influenciam a decisão de compra.

Portanto, observam-se inicialmente, dois comportamentos marcantes como decorrência da consciência ambiental, (1) a consciência ambiental ocasionando um hábito de consumo sustentável (WEBSTER, 1975; KAHLE, 1996; BEDANTE; SLONGO, 2004; GARCIA 2008; PINTO et al., 2011) e (2) a consciência ambiental ocasionando uma intenção de compra de produtos ecologicamente corretos (KAHLE, 1996; MENON; MENON, 1997; PRIEWASSER, 1999; MOTTA; ROSSI, 2003; VERMEIR; VERBEKE, 2006; MARCHAND; WALKER, 2007; PEATTIE; 2010). Com base nestas evidências, elaboraram-se as seguintes hipóteses:

\section{H1: A consciência ambiental influencia positivamente os bábitos de consumo sustentável.}

H2: A consciência ambiental influencia positivamente a intenção de compra de produtos ecológicos.

\section{Hábitos de Consumo Sustentável}

Os hábitos de consumo adotados em todo o mundo são a principal causa da deterioração ambiental (CHEN; CHAI, 2010) e a reversão deste quadro exige que os atuais padrões de produção e consumo sejam repensados (LAZZARINI; GUNN, 2007, p.7). Comportamentos de consumo cotidianos, muitas vezes são repetitivos, se tornando hábitos, práticas institucionalizadas, reforçadas entre a população (HODGSON, 1997).

Quando hábitos se tornam automáticos, certos estímulos ambientais podem ativar as respostas praticadas de forma inconsciente (JI; WOOD, 2007). Hábitos então, se tornam fortes preditores de comportamento indicando que tal comportamento é adotado sem muita deliberação e raciocínio (DANNER; AARTS; VRIES, 2008). No entanto, hábitos também são muitas vezes adotados em função das recompensas de curto prazo que substituem os 
benefícios de longo prazo (por exemplo, a possibilidade de ocorrer alguma catástrofe climática em algumas décadas) (FAIERS; COOK; NEAME, 2007).

Apesar da dificuldade de mudança dos hábitos de consumo, em função de refletirem crenças profundamente arraigadas (SEYFANG, 2004), a sua automaticidade também dependente do contexto (MARÉCHAL, 2010) e cada vez mais os consumidores vêm sendo pressionados a fazerem sua parte para salvar o planeta, consumindo produtos reciclados e eticamente produzidos (DETR, 1999).

Consumir de forma sustentávelé assumir um comportamento favorável ao meio ambiente, intelectualmente, eticamente e na prática (MOISANDER, 2007). O consumo sustentável indica que as gerações presentes se preocupam com a finitude futura dos recursos naturais (BRUNDTLAND, 1988).

Decisões individuais dependem de valores éticos, crenças, costumes, cultura e influências sociais, políticas, morais e configurações institucionais que moldam as atitudes dos consumidores, incentivando ou desencorajando comportamentos e atitudes (BERGLUND; MAT'TI, 2006). As escolhas do consumidor refletem não apenas as preferências de preços e qualidade, mas também valores sociais e morais (MAZAR; ZHONG, 2010), além de crenças individuais sobre a consequência dos hábitos de consumo adotados (AJZEN; FISHBEIN, 1980).

Portanto, o consumo sustentável implica na utilização de produtos que atendam às necessidades básicas e melhorem a qualidade de vida, reduzindo o uso de recursos naturais e materiais tóxicos, as emissões de resíduos e poluentes, de maneira que as necessidades das gerações futuras não fiquem comprometidas (OECD, 2005).

Hobson (2003) afirma que não se pode subestimar a importância de hábitos como um potencial obstáculo a uma mudança de comportamento. Sobre forma de demonstrar tal relevância, estudos têm observado uma associação significativa entre hábitos de consumo e comportamento do consumidor verde (ARSLAN; YILMAZ; AKSOY, 2012). Estas variáveis indicam que o consumo está ligado a comportamentos sociais e éticos de forma mais ampla que se pensava anteriormente (MAZAR; ZHONG, 2010).

Motta e Oliveira (2007), Silva, Ferreira e Ferreira (2009) e Nagar (2013) afirmam que estimular os hábitos de consumo sustentáveis, por meio de divulgação dos critérios ambientalmente amigáveis dos produtos e utilização de estratégias de marketing verde, influencia positivamente a aquisição de bens ecológicos. Mattar (2013) sugere que estes estímulos são fundamentais para que os consumidores mudem seus hábitos de compra.
Assim sendo, chega-se a uma terceira evidência: (3) os hábitos de consumo sustentável contribuem para a compra de produtos ecologicamente corretos (AJZEN; FISHBEIN, 1980; HOBSON, 2003; OECD, 2005; MOTTA; OLIVEIRA, 2007; SILVA; FERREIRA; FERREIRA, 2009; MAZAR; ZHONG, 2010; ARSLAN; YILMAZ; AKSOY, 2012; MATTAR, 2013; NAGAR, 2013). Portanto, a terceira hipótese foi assim definida:

H3: Os hábitos de consumo sustentável impactam positivamente a intenção de compra de produtos ecológicos

\section{Método de Pesquisa}

Este estudo caracteriza-se como descritivo, quantitativo e operacionalizado por meio de uma survey. Como instrumento de coleta de dados utilizou-se um questionário estruturado, baseado nas três dimensões propostas no estudo de Cardoso e Cairrão (2007): Hábitos de Consumo Sustentável; Intenção de Compra de Produtos Ecológicos e Consciência Ambiental. As questões podem ser visualizadas a seguir.

Quadro 1. Dimensões e variáveis propostas por Cardoso e Cairrão (2007)

\section{Hábitos de consumo sustentável}

Quando tenho que escolher entre dois produtos iguais, eu escolho sempre o que e menos prejudicial às outras pessoas e ao meio ambiente.

Não compro produtos fabricados ou vendidos por empresas que prejudicam ou desrespeitam o meio ambiente.

Faço sempre um esforço para reduzir o uso de produtos feitos de recursos naturais escassos.

Quando possível, escolho sempre produtos que causam menos poluição.

Já convenci amigos e familiares a não comprar produtos que prejudicam o meio ambiente.

Para a minha casa não compro produtos que prejudiquem o meio ambiente.

Não compro um produto quando sei dos possíveis danos que ele pode causar ao meio ambiente.

Não compro produtos e alimentos que possam causar a extinção de algumas espécies animais ou vegetais.

Procuro comprar produtos feitos em papel reciclado.

Sempre que possível, compro produtos feitos de material reciclado.

Tento comprar apenas produtos que possam ser reciclados.

Evito comprar produtos que não sejam biodegradáveis.

Compro produtos naturais porque são mais saudáveis. 
Prefiro alimentos sem fertilizantes químicos porque respeitam o meio ambiente.

Estou disposto a pagar um pouco mais por produtos e

alimentos que estejam livres de elementos químicos que prejudiquem o meio ambiente.

Quando compro produtos e alimentos à preocupação com o meio ambiente influencia a minha decisão de escolha.

Intenção de compra de produtos ecológicos

Nas suas compras o preço sempre é mais importante Prioriza compra de produtos em embalagens biodegradáveis. Compraria um produto numa embalagem reciclável em alternativa a comprar um produto similar numa embalagem não reciclável.

Estaria disposto a comprar alguns produtos (que agora compro em embalagens menores) em embalagens maiores e com menor frequência.

Compraria um produto numa embalagem pouco tradicional (por exemplo, redonda quando a maioria é quadrada) se isso se traduzisse na criação de menos resíduos sólidos (lixo).

Compraria um produto com uma embalagem menos atrativa se soubesse que todo o plástico e/ou papel desnecessário nesta embalagem tivesse sido eliminado.

\section{Consciência ambiental}

As plantas e os animais existem, basicamente, para serem utilizados pelos seres humanos.

Estamos nos aproximando do número limite de habitantes que a terra pode suportar.

Para manter uma economia saudável teremos que desenvolvêla para que o crescimento industrial seja controlado.

O planeta Terra é como uma aeronave, com espaço e recursos limitados.

Os seres humanos não precisam de se adaptar ao ambiente natural porque podem adaptar o meio ambiente as suas necessidades.

Existem limites de crescimento para além dos quais a nossa sociedade industrializada não pode expandir-se.

O equilíbrio da natureza é muito delicado e facilmente perturbado.

Quando os seres humanos interferem na natureza, isso frequentemente produz consequências desastrosas.

Os seres humanos devem viver em harmonia com a natureza para que possam sobreviver melhor.

A humanidade esta abusando seriamente do meio ambiente.

Os seres humanos têm o direito de modificar o meio

ambiente para ajustá-lo as suas necessidades.

A humanidade foi criada para dominar a natureza.

Fonte: adaptado de Cardoso e Cairrão (2007).

Observa-se no Quadro 1, as afirmações relacionadas a cada dimensão. Assim como na versão original, os itens foram mensurados por uma escala do tipo Likert de 5 pontos (1: discordo totalmente... 5: concordo totalmente).

A escala originária desenvolvida por Cardoso e Cairrão (2007) foi utilizada para pesquisar os hábitos de consumo sustentável, a intenção de compra de produtos ecológicos e a consciência ambiental de universitários de Portugal. No Brasil, este modelo foi utilizado por Gorni, Gomes e Dreher (2012), com universitários do curso de Administração.

Diferente dos trabalhos anteriormente mencionados, que analisaram a percepção dos estudantes sobre as três dimensões isoladamente, este estudo buscou analisar as relações causais entre elas, ou seja, as relações de causa e efeito dos fatores consciência ambiental e hábitos de consumo sustentável sobre a intenção de compra de produtos ecológicos. Além disso, esta pesquisa envolveu universitários de uma gama maior de cursos: Administração, Artes Visuais, Gestão Comercial, Logística, Processos Gerenciais, Pedagogia e Psicologia.

O questionário foi disponibilizado via Google docs e o link enviado por e-mail para os 736 universitários (população) dos cursos citados. Destes questionários, 182 retornaram devidamente preenchidos (correspondendo a 24,8\% do universo) e passaram a representar a amostra deste estudo.

A análise dos dados foi realizada por meio da modelagem de equações estruturais.

\section{Apresentação e Análise dos Resultados}

O primeiro passo foi a realização da purificação dos construtos, por meio da análise fatorial confirmatória (AFC) levando em consideração as cargas fatoriais de cada variável. No construto Hábitos de Consumo Sustentável (HCS) foram excluídos oito itens: HA1, HA2, HA3, HA8, HA10, HA12, HA13 e HA16. No construto Intenção de Compra de Produtos Ecológicos (ICPE) foram excluídos três itens: ICPE1, ICPE2 e ICPE3. Por fim, no construto Consciência Ambiental (CA) foram excluídos sete itens: CA1, CA2, CA3, CA4 CA5, CA6, CA11.

Todos os itens foram excluídos por apresentarem cargas fatoriais abaixo de 0,6. Esta exclusão tem por finalidade ajustar os índices a recomendação de Kline (1998), que sugere valores próximos a 0,7 . Os itens resultantes foram HA4, HA5, HA6, HA7, HA9, HA11, HA14, HA15, CA8, CA9, CA10, ICPE4, ICPE5 e ICPE6 (14 itens em três construtos). Após a exclusão dos itens, analisou-se a confiabilidade dos construtos com os demais índices resultantes; para tanto, observou-se o alpha de cronbach, a confiabilidade composta e a variância média extraída de cada construto. O resultado é apresentado na Tabela 1. 
Tabela 1. Validação dos Construtos

\begin{tabular}{c|c|c|c|}
\hline Construto & $\begin{array}{c}\text { Alpha de } \\
\text { Cronbach }\end{array}$ & $\begin{array}{c}\text { Confiabilidade } \\
\text { Composta }\end{array}$ & $\begin{array}{c}\text { Variância Média } \\
\text { Extraída }\end{array}$ \\
\hline HCS & 0,887 & 0,98 & 0,92 \\
\hline CA & 0,839 & 0,96 & 0,89 \\
\hline ICPE & 0,808 & 0,98 & 0,93 \\
\hline
\end{tabular}

Fonte: dados da pesquisa

Todos os construtos apresentam valores apropriados de alpha de cronbach, confiabilidade composta e variância média extraída conforme indicação de Hair Jr. et al. (2005), confirmando a validação dos itens em relação aos construtos (alpha de cronabach $>0,7$, confiabilidade composta $>0,7$ e variância média extraída $>0,5)$.

Após validação dos construtos, realizou-se a equação estrutural, conforme modelagem ilustrada na Figura 1.

Figura 1. Modelagem Final

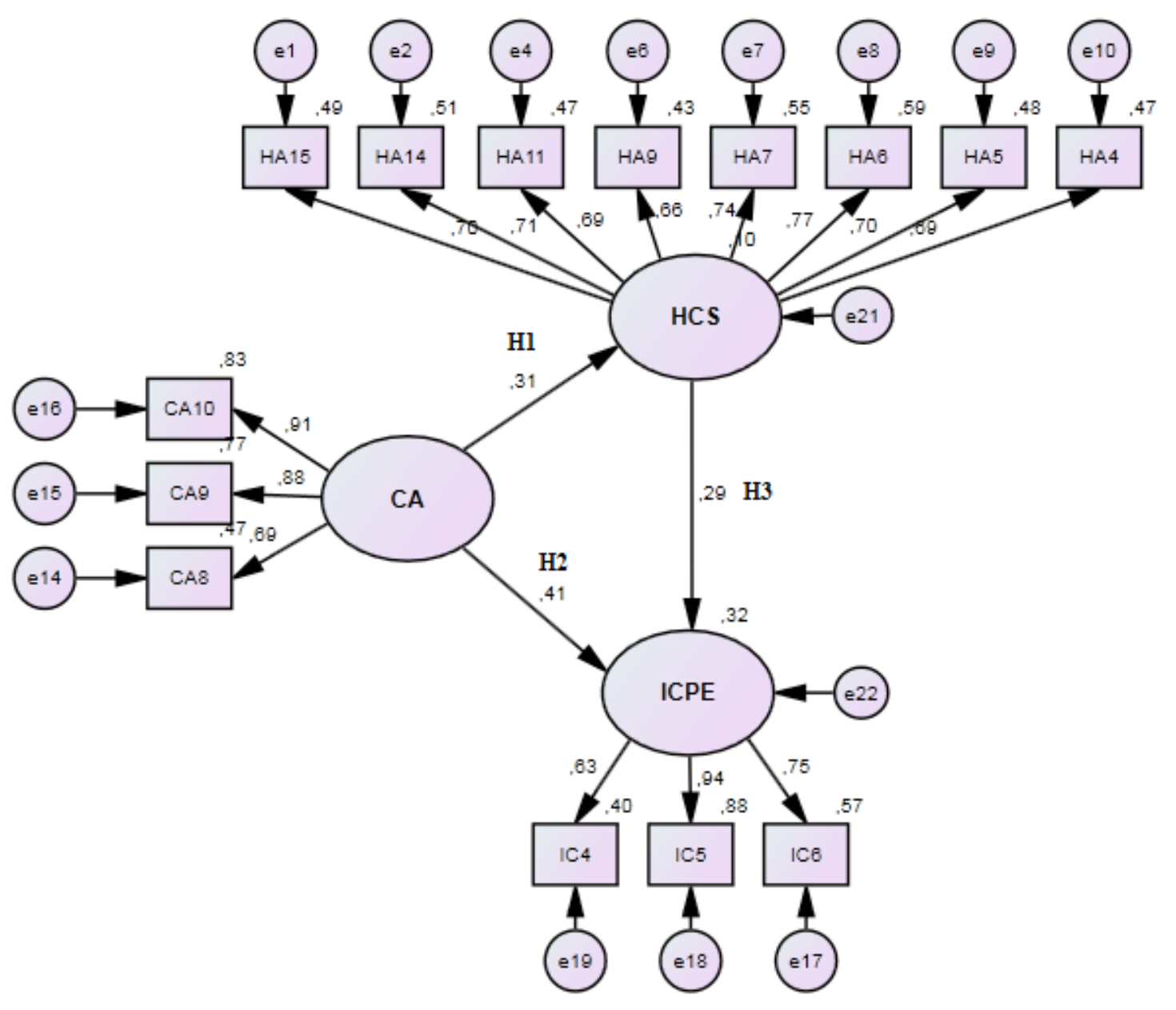

X²/GL: 2,254; GFI: 0,880; TLI: 0,909; CFI: 0,926; PNFI: 0,712; RMSEA: 0,083; SRMR: 0,063.

Fonte: dados da pesquisa 
A Figura 1 apresenta a relação entre os construtos. Observa-se que a relação entre consciência ambiental e intenção de compra de produtos ecológicos é a mais intensa $(0,41)$. A relação menos intensa é entre hábitos de consumo sustentável e intenção de compra de produtos ecológicos $(0,29)$. Isto quer dizer que a intenção de compra de produtos ecológicos é maior quando o consumidor é mais consciente sobre as questões ambientais.

As três hipóteses foram confirmadas, conforme demonstração na Tabela 2 .

Tabela 2 . Relação Entre os Construtos

\begin{tabular}{lll|cccc}
\hline \multicolumn{2}{l|}{ Relação } & & Estimação & S.E. & C.R. & P \\
\hline CA & $\grave{a}$ & HCS & 0,452 & 0,123 & 3,696 & 0,000 \\
HCS & $\grave{a}$ & ICPE & 0,253 & 0,071 & 3,568 & 0,000 \\
CA & à & ICPE & 0,512 & 0,105 & 4,896 & 0,000 \\
\hline
\end{tabular}

Fonte: dados da pesquisa

A Tabela 2 demonstra que todas as hipóteses foram confirmadas $(\mathrm{p}=0,000)$. Percebe-se assim, que a consciência ambiental influencia positivamente os hábitos de consumo sustentável (H1), corroborando com os achados de Webster (1975), Kahle (1996), Bedante e Slongo (2004), Garcia (2008) e Pinto et al. (2011). Por outro lado, Ohtomo e Hirose (2007) manifestava que consumidores ambientalmente conscientes não necessariamente possuem comportamentos ambientalmente sustentáveis, sendo esta afirmação não confirmada neste estudo.

Neste caso, chama-se a atenção o fato de que a conscientização das pessoas pode ser um primeiro passo para que elas tenham hábitos de consumo sustentáveis.

O estudo realizado demonstrou ainda que a consciência ambiental influencia positivamente a intenção de compra de produtos ecológicos (H2), respaldando os estudos de Webster (1975), Kahle (1996), Menon e Menon (1997), Priewasser (1999), Motta e Rossi (2003), Vermeir e Verbeke (2006), Marchand e Walker (2007) e Peattie (2010). Inclusive, Webster (1975), Menon e Menon (1997), Priewasser (1999) e Peattie (2010) destacaram que a consciência ambiental é considerada a principal motivadora de decisões de compra mais sustentáveis. Esta é outra evidência que destaca a importância da consciência ambiental.

Como ressaltado anteriormente, a influência da consciência ambiental sobre a intenção de compra de produtos ecológicos foi a relação mais intensa aqui pesquisada, sugerindo que a compra de produtos ecológicos possui uma relevante influência da consciência ambiental. Portanto, deve-se objetivar aumentar o nível de consciência ecológica, já que isto contribui para que o consumidor se preocupe com o impacto ambiental causado por seus atos (BEDANTE; SLONGO, 2004).

Percebe-se assim, que a ação do consumidor está relacionada com o modo de como ele pensa (BLACKWELL; MINIARD, 2000; SOLOMON, 2002). Solomon (2002) já chamava a atenção destacando que os aspectos intrínsecos são determinantes para a definição do modo de compra. Apesar de Engel, Blackwell e Miniard (2000) admitirem que os fatores psicológicos influenciarem no comportamento de compra, muitos são difíceis de serem identificados, sendo que neste trabalho, se identificou dois importantes fatores: a consciência ambiental e os hábitos de consumo.

Por fim, descobriu-se que os hábitos de consumo sustentável impactam positivamente a intenção de compra de produtos ecológicos (H3), indo ao encontro dos resultados de Ajzen e Fishbein (1980), Hobson (2003), Oecd (2005), Motta e Oliveira (2007), Ferreira e Ferreira (2009), Mazar e Zhong (2010), Arslan, Yilmaz e Aksoy (2012), Nagar (2013), Mattar (2013).

Este achado novamente indica a importância da consciência para estimular a ação, neste caso, quando consumidores possuírem hábitos de consumo sustentáveis, provavelmente, estes hábitos serão um dos motivos para impulsionar a compra de produtos sustentáveis.

\section{Considerações Finais}

A grande questão a ser resolvida atualmente é a busca pelo equilíbrio entre a qualidade de vida e a manutenção de níveis de consumo em quantidades que não provoquem a exaustão dos recursos naturais (MONT; PLEPYS, 2008). Neste estudo, partiu-se do princípio que a consciência ambiental e os hábitos de consumo sustentável impactam positivamente a intenção de compra de produtos ecológicos.

Sob esta perspectiva, realizou-se uma pesquisa quantitativa e descritiva em oito cursos superiores de uma instituição de ensino catarinense. As três hipóteses sugeridas, a consciência ambiental influencia positivamente os hábitos de consumo sustentável, a consciência ambiental influencia positivamente a intenção de compra de produtos ecológicos e os hábitos de consumo sustentável impactam positivamente a intenção de compra de produtos ecológicos, se confirmaram.

Isto indica que, além dos tradicionais critérios preço e 
conveniência, assim como outros fatores influenciadores ambientais, a perspectiva intrínseca é determinante para a escolha de produtos ecológicos, indo ao encontro dos achados de Solomon (2002), que destaca os aspectos intrínsecos como determinantes para a definição do modo de compra.

Portanto, fica a sugestão de que a compra ecológica depende também da forma como o consumidor se familiariza com o tema, já que a consciência ambiental os hábitos de consumo influenciam em sua decisão de compra. Sob uma perspectiva prática, sugere-se o desenvolvimento de campanhas e programas educativos que venham a despertar a consciência ambiental, para em momento posterior, acarretar em hábitos de intenção de compra.

\section{Sugestões e Limitações}

Aponta-se como limitação da pesquisa, o fato de a realização do estudo ter privilegiado universitários de apenas uma instituição de ensino. Como sugestão para estudos futuros, indica-se a realização de pesquisas que apontem os tipos de estímulos (educativos, sanções, publicidade, merchandising) com maior capacidade de influenciar a mudança de hábitos dos consumidores.

\section{Referências}

AJZEN, I. The theory of planned

behavior. Organizational Behavior and Human

Decision Processes, v. 50, n. 2, p. 179-211, 1991.

AJZEN, I.; FISHBEIN, M. Understanding Attitudes and Predicting Social Behaviour. Prentice-Hall, 1980.

ARSLAN, T.; YILMAZ, V.; AKSOY, H. K. Structural equation model for environmentally conscious purchasing behavior. International Journal of Environmental Research, v. 6, n. 1, p. 323-334, 2012.

BERGLUND, C.; MATTTI, S. Citizen and consumer: the dual role of individuals in environmental policy. Environmental Politics, v. 15, n. 4, p. 550-571, 2006.

BOSTRÖM, M.; KLINTMAN, M. Eco-standards, Product Labelling and Green Consumerism.

Basingstoke: Palgrave Macmillan, 2008.

BRÉCARD, D. et al. Determinants of demand for green products: An application to eco-label demand for fish in Europe. Ecological Economics, v. 69, n. 1, p. 115-125, 2009.
BRUNDTLAND, G. H. Comissão mundial sobre meio ambiente e desenvolvimento-1988. Nosso Futuro Comum, 1988.

BUTZKE, I. C.; PEREIRA, G. R.; NOEBAUER, D. Sugestão de indicadores para avaliação do desempenho das atividades educativas do sistema de gestão ambiental -SGA da Universidade Regional de Blumenau FURB. Revista Eletrônica do Mestrado em Educação Ambiental, v. 1, 2001.

CALOMARDE, J. V. Marketing ecológico. Pirámide, 2000.

CARDOSO, A. J. M.; CAIRRÃO, A. M. C. L. Os jovens universitários e o consumo sustentável: A sua influência na compra de produtos ecológicos. Revista da Faculdade de Ciência e Tecnologia, v.4, 2007.

CARVALHO, I. C. M. Qual educação ambiental? Elementos para um debate sobre educação ambiental e extensão rural. Revista Agroecologia e Desenvolvimento Rural Sustentável, Porto Alegre, v.2, n.2, 2001.

CHEN, T. B.; CHAI, L. T. Attitude towards the environment and green products: consumers' perspective. Management Science and Engineering, v. 4, n. 2, p. 27-39, 2010.

COHEN, Maurie J. The emergent environmental policy discourse on sustainable consumption. In: Exploring sustainable consumption. 2001. p. 21-37.

CONNOLLY, J.; PROTHERO, A. Sustainable consumption: consumption, consumers and the commodity discourse. Consumption, Markets and Culture, v. 6, n. 4, p. 275-291, 2003.

DANNER, Unna N.; AARTS, Henk; VRIES, Nanne K. Habit vs. intention in the prediction of future behaviour: The role of frequency, context stability and mental accessibility of past behaviour. British Journal of Social Psychology, v. 47, n. 2, p. 245-265, 2008.

DEFRA. Changing Patterns: UK Government Framework for Sustainable Consumption and Production. DEFRA, London (2003)

DETR - Department of the Environment, Transport and the Regions. Every Little bit Helps: are you doing your bit? Local Quality of Life Counts: A handbook for a menu of local indicators of sustainable development, 1999. 
DIAS, G. F.. Atividades Interdiciplinares de Educação

Ambiental. Global Editora e Distribuidora Ltda, 2013.

D’SOUZA, C. et al. Green products and corporate strategy: an empirical investigation. Society and

Business Review, v. 1, n. 2, p. 144-157, 2006.

ENGEL, James F. BLACKWELL, Roger D; MINIARD, Paul W. Comportamento do Consumidor. $8^{\mathrm{a}}$ ed. Rio de Janeiro: LTC, 2000.

FAIERS, A.; COOK, M.; NEAME, C. Towards a contemporary approach for understanding consumer behaviour in the context of domestic energy use. Energy Policy, v. 35, n. 8, p. 4381-4390, 2007.

FERREIRA, A. P. A. L. et al. A prática de sustentabilidade: um estudo bibliométrico com relação à percepção dos pesquisadores da área de administração. Revista Metropolitana de Sustentabilidade (ISSN 2318-3233), v. 5, n. 3, p. 44-60, 2015.

FRAJ, E.; MARTINEZ, E. Environmental values and lifestyles as determining factors of ecological consumer behaviour: an empirical analysis. Journal of Consumer Marketing, v. 23, n. 3, p. 133-144, 2006.

FURRIELA, R. B. Educação para o consumo sustentável. Ciclo de Palestras sobre Meio Ambiente. MEC/SEF/COEA, p. 47-55, 2001.

GARCIA, M. N. et al. Inovação no comportamento do consumidor: recompensa às empresas sócioambientalmente responsáveis. RAI - Revista de Administração e Inovação, São Paulo, v. 5, n. 2, p. 7391, 2008.

GOODWIN, N. R. The Consumer Society. Island Press, 1996.

GORNI, P. M.; GOMES, G.; DREHER, M. T. Consciência ambiental e gênero: os universitários e o consumo sustentável. Revista de Gestão Social e Ambiental, v. 6, n. 2 p. 468.

HAIR JR., J. F. et al. Análise multivariada de dados. Porto Alegre: Bookman, 2005.

HOBSON, K. Thinking habits into action: the role of knowledge and process in questioning household consumption practices. Local Environment, v. 8, n. 1, p. 95-112, 2003.

HODGSON, G. M. Economics and the return to Mecca: the recognition of novelty and emergence. Structural Change and Economic Dynamics, v. 8, n. 4, p. 399-
412, 1997.

HORNE, R. E. Limits to labels: The role of eco-labels in the assessment of product sustainability and routes to sustainable consumption. International Journal of Consumer Studies, v. 33, n. 2, p. 175-182, 2009.

JI, M. F.; WOOD, W. Purchase and consumption habits: Not necessarily what you intend. Journal of Consumer Psychology, v. 17, n. 4, p. 261-276, 2007.

KAHLE, L. R. Social values and consumer behavior: Research from the list of values. In: The psychology of values: The Ontario symposium. Mahwah, NJ: Lawrence Erlbaum Associates, 1996. p. 135-151.

KRUTER, G. E.; DE BARCELLOS, M. D.; SILVA, V. S. As Atitudes dos Consumidores em Relação ao Plástico Verde. Revista de Gestão Ambiental e Sustentabilidade-GeAS, v. 1, n. 1, p. 19-46, 2012.

KUHLMAN, T.; FARRINGTON, J. What is sustainability?. Sustainability, v. 2, n. 11, p. 3436-3448, 2010 .

LAMBIN, J. J. Marketing Estratégico. 4a ed. Madrid: McGraw- Hill, 2002.

LUCHS, M. G. et al. The sustainability liability: Potential negative effects of ethicality on product preference. Journal of Marketing, v. 74, n. 5, p. 18-31, 2010 .

MANZINI, R. et al. Assessing environmental product declaration opportunities: a reference framework. Business Strategy and the Environment, v. 15, n. 2, p. 118-134, 2006.

MARCHAND, A.; WALKER, S. Product development and responsible consumption: designing alternatives for sustainable lifestyles. Journal of Cleaner Production, v. 16, n. 11, p. 1163-1169, 2008.

MARÉCHAL, K. The economics of climate change and the change of climate in economics. Energy Policy, v. 35, n. 10, p. 5181-5194, 2007.

MARÉCHAL, K. Not irrational but habitual: The importance of "behavioural lock-in" in energy consumption. Ecological Economics, v. 69, n. 5, p. 1104-1114, 2010.

MASERA, R. Carbon sequestration dynamics in forestry projects: The CO2FIX V. 2 Model approach. In: Simposio Internacional Medición y Monitoreo de la Captura de Carbono en Ecosistemas Forestales. 2001. 
MATTAR, H. Educando para o consumo consciente: aprendizados. Brasília: Correio Brasiliense, 2013.

MAZAR, N.; ZHONG, C. Do green products make us better people?. Psychological science, 2010.

MENON, A; MENON, A. Enviropreneurial marketing strategy: the emergence of corporate environmentalism as market strategy. The Journal of Marketing, p. 51-67, 1997.

MOISANDER, J. Motivational complexity of green consumerism. International Journal of Consumer Studies, v. 31, n. 4, p. 404-409, 2007.

MONTEIRO, T. A. et al. Consciência ecológica e atitudes dos consumidores: um estudo exploratório sobre seus impactos diante de produtos e marcas. Revista de Administração da Unimep, v. 10, n. 3, p. 183-198, 2012.

MONT, O.; PLEPYS, A. Sustainable consumption progress: should we be proud or alarmed?. Journal of Cleaner Production, v. 16, n. 4, p. 531-537, 2008.

MOT'TA, S. L. S.; OLIVEIRA, B. O marketing ecológico como vantagem competitiva. REGE Revista de Gestão, v. 14, n. 2, p. 49-59, 2007.

MOT'TA, S. L. S.; ROSSI, G. B. A influência do fator ecológico na decisão de compra de bens de conveniência: um estudo exploratório na cidade de São Paulo. Revista de Administra\&ccdeil; ão da Universidade de São Paulo, v. 38, n. 1, 2003.

NAGAR, K. An empirical investigation into the influence of green advertising on brand loyalty. Journal of

Services Research, v. 13, n. 2, 2013.

OECD - Organisation for Economic Co-operation and Development. Effects of eco-labelling schemes: compilation of recent studies. OECD, Paris, 2005.

OHTOMO, S.; HIROSE, Y. The dual-process of reactive and intentional decision-making involved in eco-friendly behavior. Journal of Environmental Psychology, v. 27, n. 2, p. 117-125, 2007.

OTTMAN, J. A. Marketing Verde. São Paulo: Makron Books, 1993.

PEDERSEN, E. R.; NEERGAARD, P. Caveat emptorlet the buyer beware! Environmental labelling and the limitations of 'green'consumerism. Business Strategy and the Environment, v. 15, n. 1, p. 15-29, 2006.
PINTO, D. C. et al. Green consumer values: how do personal values influence environmentally responsible water consumption? International Journal of Consumer Studies, v. 35, n. 2, p. 122-131, 2011.

PORTILHO, F. Consumo sustentável: limites e possibilidades de ambientalização e politização das práticas de consumo. Cadernos Ebape. br, v. 3, n. 3, p. 01-12, 2005.

PRIEWASSER, R. Ecological sustainability and personal behavior: relations demonstrated by the decisionmaking process of selecting a certain transportation mean. Environmental Management and Health, v. 10, n. 3, p. 165-169, 1999.

ROBERTS, J. A. Green consumers in the 1990s: profile and implications for advertising. Journal of Business Research, v. 36, n. 3, p. 217-231, 1996.

RUMPALA, Yannick. "Sustainable consumption" as a new phase in a governmentalization of consumption. Theory and Society, v. 40, n. 6, p. 669, 2011.

SCHAEFER, A; CRANE, A. Addressing sustainability and consumption. Journal of Macromarketing, v. 25, n. 1, p. 76-92, 2005.

SCHULTZ, P. W. Inclusion with nature: The psychology of human-nature relations. In: Psychology of Sustainable Development. Springer US, 2002. p. 61-78.

SEYFANG, G. Consuming values and contested cultures: a critical analysis of the UK strategy for sustainable consumption and production. Review of Social Economy, v. 62, n. 3, p. 323-338, 2004.

SILVA, A. A.; FERREIRA, M. Z.; FERREIRA, P. A. Estratégias de marketing verde e o comportamento do consumidor: um estudo confirmatório na grande São Paulo. SIMPOI, Anais... São Paulo, 2009.

TUKKER, Arnold et al. The impacts of household consumption and options for change. Journal of Industrial Ecology, v. 14, n. 1, p. 13-30, 2010.

VEIGA, J. E. Desenvolvimento sustentável: desafio do século XXI. Rio de Janeiro: Garamond Universitária, 2006.

VIEIRA, L. M. et al. An analysis of value in an organic food supply chain. British Food Journal, v. 115, n. 10, p. 1454-1472, 2013. 
VERMEIR, I.; VERBEKE, W. Sustainable food consumption: Exploring the consumer "attitudebehavioral intention" gap. Journal of Agricultural and Environmental Ethics, v. 19, n. 2, p. 169-194, 2006.

\section{WALDMAN, M.; SCHNEIDER, D. Guia Ecológico}

Doméstico. Editora Contexto, 2000.

WEBSTER JR, F. E. Determining the characteristics of the socially conscious consumer. Journal of Consumer Research, p. 188-196, 1975.

WIEDMANN, K. et al. Tasting green: an experimental design for investigating consumer perception of organic wine. British Food Journal, v. 116, n. 2, p. 197-211, 2014.

YOUNG, et al. Sustainable consumption: green consumer behavior when purchasing products. Sustainable

Development, v. 18, n. 1, p. 20-31, 2010. 